\title{
NÍVEIS SÉRICOS DE GLOBULINAS E A INTENSIDADE DA FIBROSE HEPÁTICA EM PACIENTES COM ESQUISTOSSOMOSE MANSÔNICA
}

\author{
Henrique S. T. CORREIA, Ana Lucia C. DOMINGUES, Edmundo P. A. LOPES, \\ Clarice N. L. MORAIS, Camila SARTESCHI e Izolda M. F. MOURA
}

\begin{abstract}
RESUMO - Contexto - Tem sido descrita correlação entre os níveis séricos de globulinas e o grau de fibrose hepática nas hepatites crônicas, mas não se encontram relatos na esquistossomose mansônica. Objetivo - Avaliar os níveis séricos de globulinas e de IgG, e a intensidade da fibrose periportal mensurada pela ultrassonografia em pacientes com esquistossomose mansônica. Métodos - Entre novembro de 2006 e fevereiro de 2007, foram estudados 41 pacientes que preencheram ficha clínica e realizaram dosagens de IgG por imunoturbidimetria e de globulinas indiretamente pelo método do biureto. A ultrassonografia foi realizada por um único pesquisador, seguindo os protocolos do Cairo e de Niamey. Resultados - A média de idade foi 41 anos, sendo 25 pacientes (61\%) do sexo feminino. Dez dos 41 pacientes (24\%) apresentaram elevação dos níveis séricos de globulinas e 21 (51\%) dos de IgG. Conforme a classificação do Cairo, 21 pacientes apresentaram grau I de fibrose, 18 grau II e 2 grau III, e pela classificação de Niamey 8 apresentavam padrão C, 20 D e 13 E. Aqueles com graus II ou III de fibrose tiveram maiores níveis de IgG do que os de grau I $(P=0,047)$, assim como aqueles que apresentaram padrões $\mathrm{D}$ e $\mathrm{E}$ em relação ao $\mathrm{C}(P=0,011)$. Não houve associação entre os níveis de globulinas e o grau ou padrão de fibrose. Conclusão - Em pacientes com esquistossomose mansônica, observou-se elevação dos níveis séricos de IgG de acordo com a progressão do grau e do padrão de fibrose periportal, mas o mesmo não se observou com os níveis de globulinas. DESCRITORES - Soroglobulinas. Imunoglobulina G. Cirrose hepática. Esquistossomose mansoni.
\end{abstract}

\section{INTRODUÇÃO}

$\mathrm{Na}$ esquistossomose mansônica (EM), em sua forma hepatoesplênica (EHE), a hipertensão portal ocorre tanto em função da fibrose periportal (Symmers) como pelo hiperfluxo proveniente da veia esplênica em consequência da grande esplenomegalia ${ }^{(13,17,25)}$. Descreve-se correlação entre a intensidade da fibrose periportal e os níveis de pressão na veia porta ${ }^{(18)}$.

A biopsia hepática, a ultrassonografia (US) e, mais recentemente, marcadores biológicos e a elastografia transhepática $\left(\right.$ FibroScan $\left.{ }^{\circledR}\right)$ vêm sendo utilizados para mensurar a fibrose que se desenvolve nas doenças crônicas do fígado ${ }^{(8,15,20,22)}$.

$\mathrm{Na}$ EM, a biopsia hepática em cunha, realizada a céu aberto, é o método mais acurado para a mensuração da fibrose periportal, mas implica na realização de laparotomia. Já a biopsia por agulha nem sempre representa o verdadeiro quadro histopatológico, visto que a fibrose, apesar de ser difusa, varia de intensidade no parênquima hepático(6).
A US, através da identificação do aumento do lobo hepático esquerdo e redução do direito, do espessamento periportal, além da comprovação da grande esplenomegalia, constitui importante ferramenta na propedêutica da $\mathrm{EM}^{(4,7,19)}$. Em 1990, convenção da OMS realizada no Cairo classificou em três graus a fibrose periportal na EM, e outra convenção em 1996, em Niamey, propôs nova classificação baseada em seis padrões de A a F, que auxiliam na avaliação da morbidade ${ }^{(27,28)}$. As duas classificações apresentam grande precisão, mas requerem equipamentos sofisticados e examinadores qualificados, inferindo a necessidade de que métodos mais simples sejam implementados.

Sabe-se que as células hepáticas estreladas, também chamadas células de Ito, são as principais mediadoras da fibrose hepática e quando ativadas, se transformam em células proliferativas e contráteis, constituindo a essência da resposta fibrótica à agressão hepática ${ }^{(3,9)}$. Estas células são ativadas pelo fator de necrose tumoral alfa (TNF- $\alpha$ ), pelo fator de crescimento derivado das

Estudo desenvolvido no Ambulatório de Esquistossomose e no Laboratório Central do Hospital das Clínicas da Universidade Federal de Pernambuco, Recife, PE. Correspondência: Dr. Edmundo P.A. Lopes - Rua Irmã Maria Davi, 154 - apt.3201 - Casa Forte - 52061-070 - Recife, PE. E-mail: epalopes@uol.com.br 
plaquetas (PDGF) e por citocinas fibrogênicas, tais como TGF- $\beta$, angiotensina II e leptina ${ }^{(3,9)}$.

Acredita-se ainda que as imunoglobulinas exerçam efeito direto sobre a fibrogênese hepática e que a imunoglobulina $\mathrm{G}(\mathrm{IgG})$ regule a diferenciação e a proliferação das células estreladas ${ }^{(23)}$. Recentemente, dois artigos demonstraram correlação entre os níveis séricos de globulinas e de imunoglobulinas e o grau de fibrose hepática em pacientes com hepatites virais crônicas ${ }^{(21,26)}$.

Além das proteínas responsáveis pela fibrogênese, outras provenientes do processo de degradação da matriz extracelular, também vêm sendo utilizadas na avaliação da fibrose hepática, incluindo o ácido hialurônico, a laminina, o colágeno tipo IV, a hidroxiprolina urinária, entre outras $(2,10,11,15,20,22,29)$.

Em virtude da escassez de dados sobre marcadores de fibrose na EM, este estudo teve como objetivo avaliar os níveis séricos de globulinas e de $\mathrm{IgG}$, assim como o grau e o padrão de fibrose periportal, estabelecidos pela US, empregando tanto a classificação do Cairo como a de Niamey ${ }^{(27,28)}$.

\section{MÉTODO}

Foram avaliados pacientes com fibrose periportal relacionada à EM, atendidos no período de novembro de 2006 a fevereiro de 2007, no Ambulatório de Esquistossomose do Hospital das Clínicas (HC) da Universidade Federal de Pernambuco (UFPE). O diagnóstico da EM foi confirmado através da história clínica e pela presença da fibrose periportal à US.

Os critérios de inclusão foram: pacientes com fibrose periportal esquistossomótica de ambos os sexos com idade acima de 18 anos. Foram considerados critérios de exclusão a presença de marcadores da hepatite $\mathrm{B}$ ou C (HBsAg, anti$\mathrm{HBc}$ e anti-HCV), consumo de etanol $>210 \mathrm{~g} / \mathrm{semana}$, ou a identificação de outras doenças hepáticas coexistentes.

Após a análise dos critérios de inclusão e de exclusão, os pacientes foram esclarecidos sobre os objetivos do protocolo, convidados a participar do estudo e a assinar o termo de consentimento livre e esclarecido.

$\mathrm{O}$ protocolo de estudo foi analisado e aprovado pelo Comitê de Ética em Pesquisa do Centro de Ciências da Saúde da UFPE.

O desenho do estudo foi descritivo e incluiu 41 pacientes que apresentavam uma das seguintes formas clínicas da EM: hepatointestinal (EHI), hepatoesplênica (EHE) e hepatoesplênica pós-esplenectomia (EHE-E).

Foram preenchidas fichas com informações dos pacientes e colhido sangue em veia periférica para a realização dos exames no Laboratório Central do HC-UFPE. Os níveis séricos de IgG foram aferidos pelo método de imunoturbidimetria (Tina-quant - Roche ${ }^{\circledR}$ ) e os de globulinas foram indiretamente mensurados utilizando-se o método do biureto. Foram considerados os seguintes valores de normalidade: globulinas $=3.100$ a $3.700 \mathrm{mg} / \mathrm{dL}$ e de $\operatorname{IgG}=700$ a $1.600 \mathrm{mg} / \mathrm{dL}$.

Todos os pacientes foram submetidos, pelo mesmo examinador, ao exame ultrassonográfico com equipamento ALOKA $^{\circledR}$ SSD500 com transdutor de 3,5 MHz para avaliação do fígado e do baço, segundo protocolos estabelecidos pela OMS. A classificação do Cairo padronizou três graus para a fibrose periportal na EM de acordo com a média obtida das medidas do diâmetro externo-externo de três ramos periféricos da veia porta após a segunda ramificação: grau I $>3$ a $5 \mathrm{~mm}$; grau II $>5$ a $7 \mathrm{~mm}$ e grau III $>7 \mathrm{~mm}^{(27)}$. Já a classificação de Niamey emprega seis padrões pré-estabelecidos de apresentação da fibrose periportal, graduados de A a F, de acordo com a gravidade, sendo $\mathrm{A}$ fígado normal e $\mathrm{F}$ fibrose muito acentuada ${ }^{(28)}$.

A análise estatística foi realizada utilizando-se os softwares: SPSS for Windows, versão 12.0, para a execução dos cálculos estatísticos, elaboração e edição dos gráficos. Foram considerados estastisticamente significantes os resultados cujos níveis foram inferiores a $5 \%(P<0,05)$.

Para avaliar os níveis séricos de globulinas e de $\mathrm{IgG}$ em relação ao grau de fibrose periportal pela classificação do Cairo, utilizou-se o Teste Mann-Whitney; quando se empregou a classificação de Niamey, utilizou-se o teste de Kruskal-Wallis. Foram utilizados testes não-paramétricos, já que as curvas com as dosagens dos níveis séricos de globulinas e de IgG se afastaram da distribuição gaussiana.

\section{RESULTADOS}

As características demográficas dos 41 pacientes são as seguintes: a média de idade foi 41 anos e a mediana 48, com variação de 23 a 73 anos; 25 pacientes eram do sexo feminino (61\%) e 16 do masculino (39\%), com proporção F/M de 1,6:1.

A Tabela 1 apresenta a distribuição das formas clínicas da EM dos 41 pacientes, de acordo com o grau e o padrão de fibrose periportal, avaliados pela US, empregando-se as classificações do Cairo e de Niamey, respectivamente.

TABELA 1. Distribuição das formas clínicas de 41 pacientes com esquistossomose mansônica de acordo com o grau e o padrão de fibrose periportal avaliados pela ultrassonografia, conforme as classificações do Cairo e de Niamey, respectivamente

\begin{tabular}{lccccccc}
\hline & Total & \multicolumn{3}{c}{ Classificação do Cairo } & \multicolumn{3}{c}{ Classificação de Niamey } \\
& $(\%)$ & I & II & III & C & D & E \\
\hline EHI & $12(29)$ & 9 & 3 & 0 & 8 & 3 & 1 \\
EHE & $19(46)$ & 6 & 12 & 1 & 0 & 10 & 9 \\
EHE-E & $10(24)$ & 6 & 3 & 1 & 0 & 7 & 3 \\
Total $(\%)$ & $41(100)$ & $21(51)$ & $18(44)$ & $2(5)$ & $8(20)$ & $20(49)$ & $13(32)$ \\
\hline
\end{tabular}

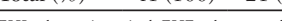

EHE-E = hepatoesplênica pós-esplenectomia

Três dos 12 (25\%) pacientes com a forma EHI, 12 dos 19 $(63 \%)$ com a forma EHE e 6 dos $10(60 \%)$ com a forma EHE-E apresentavam elevações dos níveis de IgG. Ou seja, 21 (51\%) dos 41 pacientes apresentavam níveis séricos elevados de IgG.

Um dos 12 (8\%) pacientes com a forma EHI, 6 dos 19 $(32 \%)$ com a forma EHE e 3 dos $10(30 \%)$ com a forma EHE-E apresentavam elevações dos níveis de globulinas. Ou seja, $10(24 \%)$ dos 41 pacientes apresentavam níveis séricos aumentados de globulinas.

Observou-se correlação entre os níveis séricos de IgG e os de globulinas $(\mathrm{r}=0,78)$ dos 41 pacientes com EM.

As médias e medianas dos níveis séricos de $\mathrm{IgG}$ e de globulinas dos 41 pacientes com EM podem ser observadas 
na Tabela 2 e na Figura 1. Para análise estatística, os pacientes foram distribuídos em dois grupos, I e II/III, de acordo com o grau de fibrose, avaliados pela US conforme a classificação do Cairo, em virtude da ocorrência de apenas dois pacientes com grau III. Analisando-se os resultados desta tabela, verifica-se que os pacientes com grau II/III apresentaram níveis séricos superiores de $\mathrm{IgG}$ em relação aos dos pacientes com grau I $(P=0,047)$.

TABELA 2. Médias dos níveis séricos de $\operatorname{IgG}(\mathrm{mg} / \mathrm{dL})$ e de globulinas $(\mathrm{mg} / \mathrm{dL})$ de 41 pacientes com esquistossomose mansônica, distribuídos em dois grupos, I e II/III, conforme o grau de fibrose à ultrassonografia, empregando-se a classificação do Cairo

\begin{tabular}{lcccccccc}
\hline & Fibrose & n & Média & DP* & Mínimo & Mediana & Máximo & $\boldsymbol{P \#}$ \\
\hline \multirow{2}{*}{$\begin{array}{l}\text { IgG } \\
(\mathrm{mg} / \mathrm{dL})\end{array}$} & I & 21 & 1.567 & \pm 456 & 1.016 & 1.405 & 2.579 & \\
& II e III & 20 & 1.843 & \pm 487 & 1.189 & 1.786 & 3.159 & 0,047 \\
& Total & 41 & 1.702 & \pm 486 & 1.016 & 1.600 & 3.159 & \\
\multirow{2}{*}{$\begin{array}{l}\text { Globulinas } \\
\text { (mg/dL) }\end{array}$} & I & 21 & 3.319 & \pm 590 & 2.500 & 3.200 & 4.400 & \\
& II e III & 20 & 3.565 & \pm 632 & 2.800 & 3.500 & 5.400 & 0,126 \\
& Total & 41 & 3.439 & \pm 616 & 2.500 & 3.400 & 5.400 & \\
\hline
\end{tabular}

$* \mathrm{DP}=$ desvio-padrã
\# Mann-Whirney

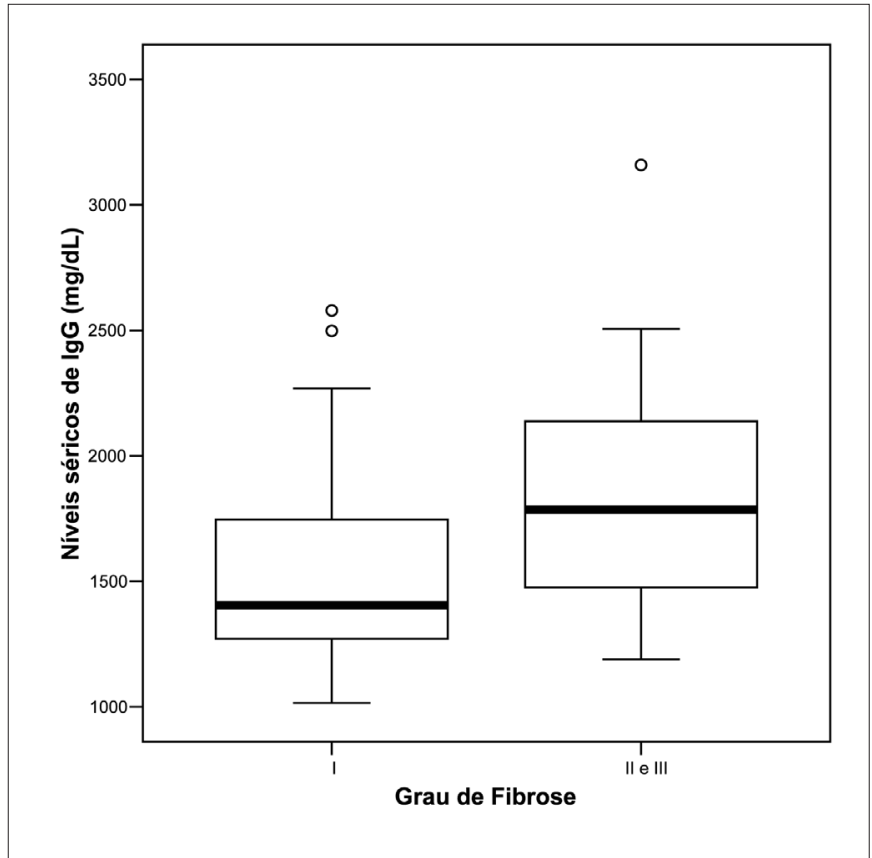

FIGURA 1. Médias dos níveis séricos de $\mathrm{IgG}(\mathrm{mg} / \mathrm{dL})$ de 41 pacientes com esquistossomose mansônica, divididos em dois grupos (I x II/III) de acordo com o grau de fibrose à ultrassonografia, empregando-se a classificação do Cairo $(P=0,047)$

$\mathrm{Na}$ Tabela 2 ainda se verifica que não houve diferenças nas médias dos níveis séricos de globulinas proporcional ao grau de fibrose avaliado pela classificação do Cairo $(P=0,126)$.

As médias e medianas dos níveis séricos de IgG e de globulinas dos 41 pacientes com EM, distribuídos em três grupos, $\mathrm{C}, \mathrm{D}$ e E, de acordo com o padrão de fibrose periportal, avaliados pela US conforme a classificação de Niamey, podem ser observadas na Tabela 3 e na Figura 2. Analisando-se os resultados desta tabela, verifica-se que os níveis séricos de IgG aumentaram conforme a evolução do padrão $\mathrm{C}$ para o padrão E de fibrose $(P=0,011)$.

TABELA 3. Médias dos níveis séricos de $\operatorname{IgG}(\mathrm{mg} / \mathrm{dL})$ e de globulinas $(\mathrm{mg} / \mathrm{dL})$ de 41 pacientes com esquistossomose mansônica, distribuídos em três grupos, C, D e E, conforme o padrão de fibrose à ultrassonografia, empregando-se a classificação de Niamey

\begin{tabular}{lcccccccc}
\hline & Fibrose & $\mathbf{n}$ & Média & DP $^{*}$ & Mínimo & Mediana & Máximo & $\boldsymbol{P}^{\#}$ \\
\hline \multirow{4}{*}{$\mathrm{IgG}$} & $\mathrm{C}$ & 8 & 1.284 & \pm 191 & 1.016 & 1.299 & 1.600 & \\
$(\mathrm{mg} / \mathrm{dL})$ & $\mathrm{D}$ & 20 & 1.741 & \pm 416 & 1.252 & 1.749 & 2.579 & 0,011 \\
& $\mathrm{E}$ & 13 & 1.898 & \pm 575 & 1.189 & 2.004 & 3.159 & \\
& Total & 41 & 1.702 & \pm 486 & 1.016 & 1.600 & 3.159 & \\
\hline \multirow{4}{*}{ Globulinas } & $\mathrm{C}$ & 8 & 3.088 & \pm 372 & 2.700 & 3.000 & 3.800 & \\
$(\mathrm{mg} / \mathrm{dL})$ & $\mathrm{D}$ & 20 & 3.480 & \pm 626 & 2.500 & 3.500 & 4.900 & 0,112 \\
& $\mathrm{E}$ & 13 & 3.592 & \pm 671 & 2.900 & 3.500 & 5.400 & \\
& Total & 41 & 3.439 & \pm 616 & 2.500 & 3.400 & 5.400 & \\
\hline
\end{tabular}

$* \mathrm{DP}=$ desvio-padrã

\# Kruskal-Wallis

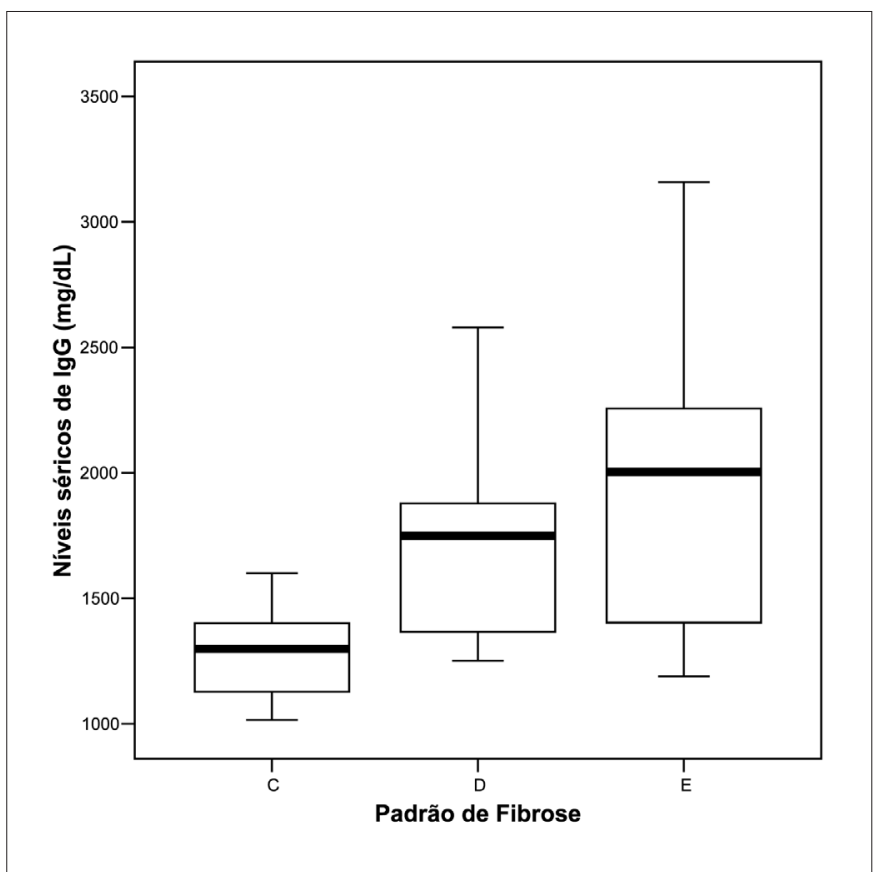

FIGURA 2. Médias dos níveis séricos de $\mathrm{IgG}(\mathrm{mg} / \mathrm{dL})$ de 41 pacientes com esquistossomose mansônica, divididos em três grupos (C, D e E), de acordo com o padrão de fibrose à ultrassonografia, empregando-se a classificação de Niamey $(P=0,011)$

Os resultados do teste de comparações múltiplas revelaram diferenças entre os níveis séricos de IgG dos 8 pacientes com padrão C e os 20 com padrão $\mathrm{D}(P=0,019)$, bem como entre os níveis dos 8 pacientes com padrão $\mathrm{C}$ e os 13 com padrão $\mathrm{E}(P=0,004)$. Não se observaram diferenças nas médias dos níveis séricos de $\mathrm{IgG}$ entre os 20 pacientes com padrão $\mathrm{D}$ e os 13 com padrão E $(P=0,327)$. Dessa forma, percebe-se que os 8 pacientes com pouca fibrose (padrão C) apresentaram 
valores menores de $\mathrm{IgG}$ em relação aos dos 33 com muita fibrose (padrão D e E).

$\mathrm{Na}$ Tabela 3, ainda se verifica que não houve diferenças nas médias dos níveis séricos de globulinas proporcional ao padrão de fibrose avaliado pela classificação de Niamey $(P$ $=0,112$ ).

\section{DISCUSSÃO}

Casos graves de EM continuam ocorrendo atualmente, visto que $70 \%$ dos pacientes neste estudo apresentaram a forma EHE, muitos com antecedentes de hemorragia digestiva e de esplenectomia, associada aos graus e padrões mais avançados de fibrose hepática (Tabela 1). Com efeito, métodos propedêuticos para aferição precoce da intensidade da fibrose periportal, que sejam simples e possam ser levados ao campo, para tratamento destes pacientes e prevenção primária dos episódios de sangramento, persistem sendo necessários.

A elastografia transhepática $\left(\right.$ FibroScan $\left.{ }^{\circledR}\right)$, método recente que avalia a rigidez do parênquima do fígado, tem revelado grande correlação com a histopatologia na mensuração da intensidade da fibrose ${ }^{(8)}$. Apresenta as mesmas vantagens da US por não ser invasivo além de avaliar várias porções do parênquima e poder ser empregado repetidamente no seguimento dos pacientes. Mas, sua principal vantagem é ser menos dependente do examinador ou menos subjetivo do que a US, assim, podendo ser executado por paramédicos com treinamento mais simples e sem a necessidade de longa experiência ${ }^{(8)}$. O aparelho para realização da elastografia, entretanto, ainda apresenta elevado custo, além de não se dispor de dados a respeito de sua precisão na avaliação da fibrose periportal na EM.

Com a intenção de contornar as desvantagens da biopsia do fígado, mormente por constituir método invasivo e estar sujeito a erros de amostragem, nos últimos anos algumas substâncias envolvidas na produção ou degradação do colágeno vêm sendo avaliadas, tanto isoladamente como em conjunto, compondo os chamados índices, na avaliação da fibrose que se desenvolve nas doenças crônicas do fígado ${ }^{(9,14,16,20)}$.

Também tem sido descrita correlação entre os níveis séricos de globulinas e de imunoglobulinas com o grau de fibrose hepática, avaliado histologicamente nas hepatites crônicas pelos vírus B e $\mathrm{C}^{(20,25)}$. SHEN et al. ${ }^{(23)}$ verificaram que as células hepáticas estreladas possuem receptores para o fragmento $\mathrm{Fc}$ da $\mathrm{IgG}$ e também demonstraram em ratos que a IgG estimula diretamente, de forma dose dependente, a atividade proliferativa das células de Ito $^{(23)}$.

Neste estudo foi observado que os níveis séricos de IgG foram maiores nos pacientes com graus de fibrose periportal mais avançados, II e III (Tabela 2), e naqueles com padrões mais acentuados, D e E (Tabela 3). Deve-se admitir, conforme demonstrado nas hepatites virais, que a IgG desempenhe certo papel no processo fibrogênico também na EM, e possa ser utilizada como marcador da intensidade da fibrose periportal.

Vale lembrar que os anticorpos contra os ovos e os vermes adultos, especialmente da classe $\operatorname{IgG}$, cursam com níveis séricos mais elevados naqueles pacientes com EM que apresentam fibrose periportal e esplenomegalia ${ }^{(24)}$. Além disso, a carga parasitária encontra-se relacionada com a evolução para as diversas formas da EM. Foi demonstrada em pacientes jovens, até 30 anos, correlação entre carga parasitária e grau de fibrose periportal e tamanho do baço e do fígado ${ }^{(1,10)}$. Assim, quanto mais vermes e ovos maior será a esplenomegalia, proporcionando maior produção de anticorpos ( $\operatorname{IgG})$, e maior intensidade da fibrose periportal. De fato, no estudo ora descrito, constatou-se que, entre 29 pacientes com EHE ou EHE-E, 18 (62\%) apresentavam níveis séricos de IgG acima do limite superior da normalidade, enquanto que esta alteração foi observada em apenas $3(25 \%)$ dos 12 com EHI.

Nesta série, entretanto, não foi verificada associação entre os níveis séricos de globulinas, nem com o grau, nem com o padrão de fibrose periportal. Uma das explicações para este achado poderia ser o tamanho da amostra, que deve ter sido suficiente para revelar elevação significativa dos níveis de IgG paralela ao aumento do grau e do padrão da fibrose periportal, mas foi insuficiente para evidenciá-la entre a fibrose e os níveis de globulinas. Outra possibilidade seria que as outras globulinas, além das imunoglobulinas, não se elevariam acompanhando o avanço da intensidade da fibrose.

Adicionalmente, no artigo sobre a fibrose na hepatite $\mathrm{C}$, descreveu-se correlação da fibrose hepática não apenas com os níveis de IgG, mas também com os de IgA e com o nível sérico total das imunoglobulinas ${ }^{(24)}$. Já no estudo sobre a fibrose na hepatite B, observou-se correlação tanto com os níveis de IgG como com os de globulinas e com a contagem de plaquetas no sangue periférico, mas não foi detectada com os níveis de $\operatorname{Ig} \mathrm{A}^{(19)}$. Desta forma, SCHMILOVITZ-WEISS et al. ${ }^{(21)}$ propuseram um índice de aumento da fibrose, ou seja, para cada $330 \mathrm{mg} / \mathrm{dL}$ de globulina deveria ocorrer aumento de 0,5 ponto no grau de fibrose hepática pela classificação de DESMET et al. ${ }^{(5)}$

Outra enfermidade hepática que cursa, caracteristicamente, com níveis elevados de imunoglobulinas é a hepatite autoimune, por conta da grande produção dos autoanticorpos. Neste estudo não foram pesquisados os autoanticorpos para afastar sorologicamente a possibilidade de doença hepática por autoimunidade, mas a fibrose observada através da US nas doenças autoimunes é do tipo difusa, diferente da fibrose de Symmers da $\mathrm{EM}^{(1,4,7,10)}$. O aspecto ultrassonográfico da fibrose periportal é facilmente reconhecido por profissionais experientes e que trabalham em áreas endêmicas.

Recentemente, em pacientes com EM, foi descrita correlação entre a fibrose periportal e a contagem de plaquetas, assim como com o índice APRI, que utiliza o nível sérico de AST dividido pelo número de plaquetas, para avaliação da fibrose ${ }^{(12)}$.

Estes índices vêm sendo cada vez mais e melhor estudados, e deverão se tornar em futuro próximo grande ferramenta não-invasiva na mensuração da fibrose hepática, sobretudo por poderem ser repetidos no seguimento dos pacientes.

Em conclusão, estes dados sugerem que os níveis séricos de IgG poderão constituir novo marcador sérico para aferição do grau e do padrão de fibrose periportal na EM. Mais estudos, envolvendo maior número de pacientes, precisam 
ser desenvolvidos para confirmar estes resultados, bem como reavaliar a relação entre os níveis séricos das globulinas e a intensidade de fibrose. Faz-se necessária ainda a criação de um índice específico que envolva mais de um marcador sérico, incluindo também os níveis de IgG, para mensuração da fibrose periportal que ocorre na EM.

Correia HST, Domingues ALC, Lopes EPA, Morais CNL, Sarteschi C, Moura IMF. Serum globulin levels and intensity of hepatic fibrosis in patients with mansonic schistosomiasis. Arq Gastroentero. 2009;46(3):194-8.

ABSTRACT - Background - A correlation between the levels of serum globulins and the hepatic fibrosis degree in chronic hepatitis was described, but reports in schistosomiasis mansoni have not been found. Objective - To evaluate the serum globulins and IgG levels, and periportal fibrosis intensity measured by ultrasound in patients with schistosomiasis mansoni. Methods - Between November, 2006 and February 2007, 41 patients which were eligible, filled them a questionnaire and had their levels of serum IgG measured by immunoturbidimetry and globulins indirectly measured by the Biuret method. The ultrasound was carried out by a single researcher, according to the Cairo and Niamey protocols. Results - The average age was 41 years old and 25 female patients (61\%). Ten patients (24\%) from 41 showed serum globulins levels raised and $21(51 \%)$ presented elevated IgG levels. According to the Cairo classification, 21 patients showed grade I of fibrosis, 18 grade II and 2 grade III; and by the Niamey classification 8 showed standard C, $20 \mathrm{D}$, and $13 \mathrm{E}$. Those with grade II or III of fibrosis had higher IgG levels than the ones with grade I $(P=0,047)$, as well as those who showed standards $\mathrm{D}$ and $\mathrm{E}$ as compared to $\mathrm{C}(P=0,011)$. There was no association between the globulins levels and the intensity of fibrosis. Conclusion - In patients with schistosomiasis mansoni, an increase of the $\mathrm{IgG}$ serum levels was observed according to the progression from periportal fibrosis intensity, but the same was not founded with globulins levels.

HEADINGS - Serum globulins. Immunoglobulin G. Liver cirrhosis. Schistossomiasis mansoni.

\section{REFERÊNCIAS}

1. Abdel-Wahab MF, Esmat G, Narooz SI, Yosery A, Struewing JP, Strickland GT. Sonografic studies of school children in a village endemic for Schistosoma mansoni. Trans R Soc Trop Med Hyg. 1990;84:69-73.

2. Aguiar JL, Domingues ALC, Aguiar SM, Kelner S. Hidroxiprolina urinária na fase hepatoesplênica da esquistossomose mansônica. An Fac Med Univ Fed Pernamb. 1997;42:38-41

3. Bataller R, Brenner DA. Liver fibrosis. J Clin Invest. 2005;115:209-18.

4. Cerri GG, Alves VA, Magalhães A. Hepatosplenic schistosomiasis mansoni. Radiology. 1984;153:777-80.

5. Desmet VJ, Gerber M, Hoofnagle JH, Manns M, Scheuer PJ. Classification of chronic hepatitis: diagnosis, grading and staging. Hepatology. 1994;19:1513-20.

6. Dimmette RM. Liver biopsy in clinical schistosomiasis; comparison of wedge and needle types. Gastroenterology. 1955;29:219-34.

7. Domingues ALC, Lima ARF, Dias HS, Leão GC, Coutinho A. An ultrasonographic study of liver fibrosis in patients infected with Schistosoma mansoni in north-east Brazil. Trans R Soc Trop Med Hyg. 1993;87:555-8.

8. Foucher J, Chanteloup E, Verginol J, Castera L, Le Bail B, Adhoute X, Bertet J, Couzigou P, De Lédinghen V. Diagnosis of cirrhosis by transient elastography (FibroScan): a prospective study. Gut. 2006;55:403-8.

9. Friedman SL. Stellate cells: a moving target in hepatic fibrogenesis. Hepatology. 2004:40:1041-3.

10. Homeida MA, Ahmed S, Dafalla A, Suliman S, Eltom I, Nash T, Bennett JL. Morbidity associated with Schistosoma mansoni infection as determined by ultrasound: a study in Gezira, Sudan. Am J Trop Med Hyg. 1988;39:196-201.

11. Kopke-Aguiar LA, Martins JR, Passerotti CC, Toledo CF, Nader HB, Borges DR. Serum hyaluronic acid as a comprehensive marker to assess severity of liver disease in schistosomiasis. Acta Trop. 2002;84:117-26.

12. Lambertucci JR, Silva LC, Antunes CM. Aspartate aminotransferase to platelet ratio index and blood platelet count are good markers for fibrosis evaluation in schistosomiasis mansoni. Rev Soc Bras Med Trop. 2007;40:559.

13. Maia MD, Lopes EPA, Ferraz AAB, Barros FMR, Domingues, ALC, Ferraz EM Evaluation of splenomegaly in the hepatosplenic form of mansoni schistosomiasis. Acta Trop. 2007;101:183-6.

14. Morais CNL, Montenegro SML, Abath FGC, Souza W, Jucá NT, Domingues ALC, Lopes EPA, Melo WG, Carvalho BM. Preliminar evaluation of cytokines in the hepatitis $\mathrm{C}-$ schistosomiasis co-infection. Mem Inst Oswaldo Cruz. 2006;101:353-4.

15. Murawaki Y, Ikuta Y, Okamoto K, Koda M, Kawasaki H. Diagnostic value of serum markers of connective tissue turnover for predicting histological staging and grading in patients with chronic hepatitis C. J Gastroenterol. 2001:36:399-406.

16. Oliveira LFA, Moreno EC, Gazzinelli G, Martins-Filho AO, Silveira MAS Gazzinelli A, Malaquias LCC, LoVerde P, Leite PM, Correa-Oliveira R. Cytokine production associated with periportal fibrosis during chronic schistosomiasis mansoni in humans. Infect Immun. 2006;74:1215-21.

17. Raia S, Mies S, Alfieri JF. Portal hypertension in mansonic schistosomiasis. World J Surg. 1991;15:176-87.

18. Richter J, Monteiro Eda S, Braz RM, Abdalla M, Abdel-Rahim IM, Fano U, Huntgeburth U, Feldmeier H. Sonographic organometry in Brazilian and Sudanese patients with hepatosplenic schistosomiasis mansoni and its relation to the risk of bleeding from oesophageal varices. Acta Trop. 1992;51:281-90.

19. Richter J, Domingues ALC, Barata CH, Prata AR, Lambertucci JR. Report on the second satellite symposium on ultrasound in schistosomiasis. Mem Inst Oswaldo Cruz. 2001;96:151-6.

20. Rossi E, Adams LA, Bulsara M, Jeffrey GP. Assessing liver fibrosis with serum marker models. Clin Biochem Rev. 2007;28:3-10.

21. Schmilovitz-Weiss H, Tovar A, Halpern M, Sulkes J, Braun M, Rotman Y, Tur-Kaspa R, Ben-Ari Z. Predictive value of serum globulin levels for the extent of hepatic fibrosis in patients with chronic hepatitis B infection. J Viral Hepat. 2006;13:671-7.

22. Sebastiani G, Alberti A. Non invasive fibrosis biomarkers reduce but not substitute the need for liver biopsy. World J Gastroenterol. 2006;12:3682-94.

23. Shen H, Zhang M, Kaita K, Minuk GY, Rempel J, Gong Y. Expression of Fc fragment receptors of immunoglobulin $\mathrm{G}$ (Fc gammaRs) in rat hepatic stellate cells. Dig Dis Sci. 2005;50:181-7.

24. Silveira AM, Bethony J, Gazzinelli A, Kloos H, Fraga LA, Alvares MC, Prata A, Guerra HL, Loverde PT, Correa-Oliveira R, Gazzinelli G. High levels of IgG4 to Schistosoma mansoni egg antigens in individuals with periportal fibrosis. Am J Trop Med Hyg. 2002;66:542-9.

25. Vezozzo DC, Farias AQ, Cerri GG, Da Silva LC, Carrilho FJ. Assessment of portal hemodynamics by Doppler ultrasound and of liver morphology in the hepatosplenic and hepatointestinal forms of schistosomiasis mansoni. Dig Dis Sci. 2006:51:1413-9.

26. Watt K, Uhanova J, Gong Y Kaita K, Doucette K, Pettigrew N, Minuk GY Serum immunoglobulins predict the extent of hepatic fibrosis in patients with chronic hepatitis C virus infection. J Viral Hepat. 2004;11:251-6.

27. World Health Organization. Proposal for a practical guide to the standardized use of ultrasound in the assessment of pathological changes. Meeting on Ultrasonography in Schistosomiasis. 1-4 October, 1990. Cairo, Egypt; TDR/SCH/ Ultrason. 1991.

28. World Health Organization. Ultrasound in schistosomiasis. A practical guide to the standardized use of ultrasonography for the assessment of schistosomiasisrelated morbidity. Second International Workshop. 22-26 October, 1996. Niamey, Niger; 1996.

29. Wyszomirska RM, Nishimura NF, Almeida JR, Yamanaka A, Soares EC. High serum laminin and type IV collagen levels in schistosomiasis mansoni. Arq Gastroenterol. 2005;42:221-5. 